'Servicio de Urgencia. Clínica Las Condes, Santiago, Chile. 25ubdirección de Investigación, Dirección Académica. Clínica Las Condes, Santiago, Chile. ${ }^{3}$ Sección de Coloproctología, Departamento de Cirugía. Clínica Las Condes, Santiago, Chile. ${ }^{4}$ Becada de Medicina Interna, Universidad de los Andes, Santiago Chile. ${ }^{5}$ Servicio de Gastroenterología, Clínica Las Condes, Santiago, Chile.

Recibidio el 20 de diciembre de 2014, aceptado el 2 de junio de 2015.

Correspondencia a: Dr. Rodrigo Quera P. Lo Fontecilla 441, Las Condes, Santiago. Teléfono: 26108749 rquera@clc.cl

\section{Desarrollo de neoplasia en pacientes con enfermedad inflamatoria intestinal}

\author{
LITAL MEYER ${ }^{1}$, DANIELA SIMIAN ${ }^{2}$, UDO KRONBERG ${ }^{3}$, CAMILA ESTAY $^{4}$, \\ JAIME LUBASCHER ${ }^{5}$, CAROLINA FIGUEROA ${ }^{5}$, RODRIGO QUERA ${ }^{5}$
}

\section{Development of malignant tumors in patients with inflammatory bowel disease}

Background: The chronic inflammation of the intestinal mucosa, the extra-intestinal manifestations of the disease and the immunosuppressive treatment of inflammatory bowel disease may increase cancer risk. Aim: To report the demographic and clinical features of patients with IBD who developed a malignant tumor. Material and Methods: Retrospective analysis of an IBD patient registry of a private clinic, diagnosed between 1976 and 2014. Results: 437 subjects were included, aged 15-88 years (58\% women). Seventy two percent of patients had ulcerative colitis. The median time of follow up was 6 years. Ten patients (2.3\%) developed a malignant tumor. In four, the tumor could be related to IBD (two colorectal cancers, one cholangiocarcinoma and one chronic myeloid leukemia (CML)). Two of 45 patients treated with biological therapy developed a tumor (CML and hypernephroma). Three of 170 patients on immunosuppressive treatment developed tumors. Only one had a tumor possibly related with the use of azathioprine (non-melanoma skin cancer). In only two patients, the treatment was changed at the time of their cancer diagnosis, from immunosuppressive medications to mesalamine. Conclusions: Only a small proportion of these patients with IBD developed a malignant tumor. The treatment of IBD has to be determined by the severity of the disease and not by the fear of developing a neoplasia. Following recommendations is fundamental to decrease the possibility of developing this complication.

(Rev Med Chile 2015; 143: 834-840)

Key words: Biological therapy; Immunosuppressive Agents; Inflammatory Bowel Diseases; Neoplasms.

\footnotetext{
L
} a enfermedad inflamatoria intestinal (EII) es un grupo de condiciones asociadas a la presencia de inflamación crónica del tracto gastrointestinal. Las dos entidades más importantes son la colitis ulcerosa (CU) y la enfermedad de Crohn (EC). Se ha señalado que pacientes con EII presentan un mayor riesgo de desarrollar neoplasias. Esto se debería, en parte, por la inflamación crónica de la mucosa intestinal, pero también secundario a manifestaciones extraintestinales de la enfermedad y al tratamiento inmunosupresor que reciben estos pacientes ${ }^{1-3}$.
La EII se ha asociado a tumores malignos gastrointestinales, destacando el cáncer colorrectal (CCR) y el colangiocarcinoma, este último asociado a colangitis esclerosante primaria $(\mathrm{CEP})^{1,4}$. Se ha descrito también el adenocarcinoma de intestino delgado, cáncer en el reservorio y cáncer perianal $^{5}$. La EII también se ha relacionado a neoplasias extraintestinales, destacando el linfoma, cáncer de piel, carcinoma urotelial y tumores neuroendocrinos ${ }^{1,4,6,7}$.

En las últimas décadas, el tratamiento de la EII ha sido optimizado con el uso de inmuno- 
supresores (azatioprina [AZT], 6-mercaptopurina $[6-\mathrm{MP}]$ y metotrexato) y agentes biológicos (anticuerpos anti-factor de necrosis tumoral- $\alpha$ [anti-TNF $\alpha$ ] como son infliximab, adalimumab y golimumab y antintegrinas como natalizumab y vedolizumab $)^{8,9}$. En la actualidad, es claro que la terapia biológica puede ser altamente efectiva en muchos pacientes con EII, especialmente en aquellos cuya enfermedad ha probado ser refractaria a la terapia tradicional (mesalazina, esteroides e inmunosupresores) $)^{1,8-10}$. Con el tiempo, los agentes biológicos no sólo están siendo utilizados en un mayor grupo de pacientes, sino que están siendo introducidos precozmente y por períodos más prolongados.

Aunque los potenciales beneficios terapéuticos obtenidos por los inmunosupresores y la terapia biológica han sido demostrados en diferentes estudios, existe la preocupación de que estos fármacos pueden estar asociados al desarrollo de infecciones y neoplasias, siendo uno de los principales obstáculos para su uso ${ }^{11,12}$.

Sumado a esto, muchos integrantes del equipo médico que trabajan en el área de la EII no están familiarizados con los tipos específicos de neoplasias que pueden ser promovidos por estos fármacos y la magnitud del riesgo asociado a su uso. Esta falta de conocimiento puede llevar a cambiar la estrategia terapéutica en estos pacientes, difiriendo el uso de la terapia más óptima y alejando la posibilidad de una evolución favorable.

Nuestro objetivo es describir las características demográficas y clínicas de los pacientes con EII que han desarrollado neoplasia, a partir de un registro de pacientes con EII de nuestro servicio, señalando su relación con el uso de inmunosupresores o terapia biológica y las modificaciones realizadas en el tratamiento posterior al diagnóstico de neoplasia.

\section{Material y Métodos}

Estudio descriptivo retrospectivo de una cohorte histórica de pacientes con EII de Clínica Las Condes diagnosticados entre los años 1976 y 2014. Todos los pacientes cumplen con criterios diagnósticos clínicos, radiológicos, endoscópicos e histológicos de EC o $\mathrm{CU}^{13,14}$.

Cada gastroenterólogo tratante completó en el registro los datos de los pacientes con diagnóstico de EII, atendidos entre abril de 2012 y octubre de
2014. Tanto el registro de EII como este estudio en particular fueron aprobados por el Comité de Ética de la institución.

El registro incluye datos demográficos y clínicos de la enfermedad: años de enfermedad, edad al diagnóstico, tabaquismo, localización de la enfermedad, tratamiento utilizado en cada paciente, hospitalizaciones por crisis de EII, cirugías relacionadas a EII y eventos adversos asociados a cada fármaco. Tanto la CU como la EC fueron clasificadas en extensión y fenotipo según la Clasificación de Montreal ${ }^{15}$. En relación a las neoplasias, se registró el tratamiento médico previo a su diagnóstico, el tipo de neoplasia desarrollada, el año de diagnóstico, el tiempo transcurrido entre el diagnóstico de la EII y el desarrollo de neoplasia y el tratamiento recibido para la EII posterior a la neoplasia.

Se realizó un análisis descriptivo de las variables categóricas a través de frecuencia absoluta y porcentual. Las variables continuas se describieron con mediana e intervalo ya que su distribución no fue paramétrica. Se utilizó una tabla para mostrar la información referente a la neoplasia en cada paciente.

\section{Resultados}

\section{Registro de EII}

Entre abril de 2012 y octubre de 2014 se habían enrolado 437 pacientes con EII, 58\% mujeres, con una mediana de edad de 37 años (intervalo: $15-88$ años) al momento del análisis de los datos. Del total de pacientes, $315(72 \%)$ presentaban diagnóstico de CU, 111 (25\%) EC y 11 (3\%) EII no clasificable. La mediana de años de enfermedad/ seguimiento fue de 6 años (i: 1-33 años) para CU y de 6 años (i: 1-38 años) en EC. La Clasificación de Montreal de los pacientes del registro se presenta en la Tabla 1.

\section{Neoplasia}

Diez pacientes $(2,3 \%)$ habían desarrollado una neoplasia, seis hombres y cuatro mujeres. La mitad de ellos con diagnóstico de CU. En relación a la edad de diagnóstico de la EII, nueve pacientes fueron diagnosticados antes de los 40 años (tres pacientes $\leq 20$ años, seis entre 21-40 años y uno sobre los 40 años). La mediana de años de enfermedad al momento del diagnóstico de la neoplasia fue de 7 años (i: 1-31 años). 
En relación al hábito tabáquico, ningún paciente fumaba al momento del diagnóstico de cáncer y sólo un paciente era ex fumador, hábito que suspendió al momento del diagnóstico de la

Tabla 1. Clasificación de Montreal de los pacientes con Ell incluidos en el registro

\begin{tabular}{|lcc|}
\hline $\begin{array}{l}\text { Clasificación de } \\
\text { Montreal }\end{array}$ & $\begin{array}{c}\text { CU } \\
\mathbf{n = 3 1 5}(\%)\end{array}$ & $\mathbf{n}=\mathbf{1 1 1}(\%)$ \\
\hline Extensión CU & & \\
E1 Proctitis & $97(31)$ & \\
E2 Colitis Izquierda & $64(20)$ & \\
E3 Colitis Extensa & $154(49)$ & \\
Localización EC & & \\
L1 Íleon & $35(32)$ \\
L2 Colon & $45(40)$ \\
L3 lleocolónica & & $29(26)$ \\
L4 Gl superior & & $4(4)$ \\
Fenotipo EC & \\
B1 inflamatorio & & $90(81)$ \\
B2 estenosante & $11(10)$ \\
B3 fistulizante & $9(8)$ \\
Perianal & $31(28)$ \\
\hline
\end{tabular}

CU: colitis ulcerosa; EC: enfermedad de Crohn.
EII. Las características clínicas de los 10 pacientes que desarrollaron una neoplasia se presentan en la Tabla 2.

De los 10 pacientes que desarrollaron neoplasia posterior al diagnóstico de la EII, en cuatro podría estar relacionada a la patología de base (dos CCR, un colangiocarcinoma y una leucemia mieloide crónica (LMC)). La paciente que desarrolló colangiocarcinoma era una paciente de 35 años, con diagnóstico de CU de 10 años de evolución, sin adherencia al tratamiento con mesalazina, la cual fue diagnosticada con una CEP en conjunto con la neoplasia, con una sobrevida de 18 meses posterior al diagnóstico del colangiocarcinoma.

Sólo en una paciente el desarrollo de la neoplasia podría estar relacionado al tratamiento farmacológico utilizado para la EII. Se trata de una paciente joven, con diagnóstico de $\mathrm{CU}$ a los 16 años que desarrolló un cáncer de piel no-melanoma (CPNM) como evento adverso al uso de AZT en dosis adecuada por 12 años.

Por último, cinco pacientes desarrollaron neoplasias que no estarían asociadas a la EII en sí o al tratamiento utilizado en esos pacientes. Dos de ellos estaban en tratamiento con mesalazina, dos

Tabla 2. Características demográficas, clínicas y de tratamiento de los pacientes que desarrollaron neoplasia

\begin{tabular}{|c|c|c|c|c|c|c|c|}
\hline IP & $\begin{array}{l}\text { Edad* } \\
\text { (años) }\end{array}$ & Sexo & EII & Neoplasia & $\begin{array}{c}\text { Años Ell al } \\
\text { diagnóstico de } \\
\text { neoplasia }\end{array}$ & $\begin{array}{l}\text { Terapia Ell al } \\
\text { diagnóstico de } \\
\text { neoplasia }\end{array}$ & $\begin{array}{c}\text { Modificación } \\
\text { terapia } \\
\text { post-neoplasia }\end{array}$ \\
\hline 1 & 75 & $\mathrm{~F}$ & EC & Cáncer colorrectal & Al diagnóstico & Mesalazina & No \\
\hline 2 & 66 & M & $C U$ & Cáncer colorrectal & 31 & $\begin{array}{l}\text { Mesalazina } \\
\text { irregular }\end{array}$ & $\begin{array}{c}\text { Sin tratamiento } \\
\text { (proctocolectomía) }\end{array}$ \\
\hline 3 & 32 & $\mathrm{~F}$ & CU & Colangiocarcinoma & 10 & $\begin{array}{l}\text { Mesalazina } \\
\text { irregular }\end{array}$ & $\begin{array}{c}\text { No } \\
\text { (mala adherencia) }\end{array}$ \\
\hline 4 & 41 & $\mathrm{~F}$ & CU & Leucemia mieloide crónica & 7 & Adalimumab & Sí (Mesalazina) \\
\hline 5 & 32 & $\mathrm{~F}$ & $\mathrm{CU}$ & Cáncer piel no-melanoma & 16 & Azatioprina & Sí (Mesalazina) \\
\hline 6 & 30 & $\mathrm{~F}$ & $\mathrm{CU}$ & Linfoma Hodgkin & 1 & Mesalazina & No \\
\hline 7 & $\begin{array}{l}47 \\
44\end{array}$ & $\mathrm{~F}$ & EC & $\begin{array}{l}\text { NIE I } \\
\text { Cáncer de mama }\end{array}$ & $\begin{array}{l}8 \\
5\end{array}$ & $\begin{array}{l}\text { Mesalazina } \\
\text { Mesalazina }\end{array}$ & $\begin{array}{l}\text { No } \\
\text { No }\end{array}$ \\
\hline 8 & 49 & M & EC & Cáncer papilar de tiroides & 29 & Azatioprina & No \\
\hline 9 & 26 & M & EC & Hipernefroma & 3 & Infliximab & No \\
\hline 10 & 24 & M & EC & Seminoma & 6 & Azatioprina & No \\
\hline
\end{tabular}

*Edad al diagnóstico de la neoplasia. Ell: Enfermedad Inflamatoria Intestinal; F: Femenino; M: Masculino; EC: Enfermedad de Crohn; CU: Colitis ulcerosa. Neoplasias relacionadas a la EII; Neoplasias asociadas al tratamiento; Otras neoplasias. IP: identificación paciente; NIE: neoplasia intraepitelial. 


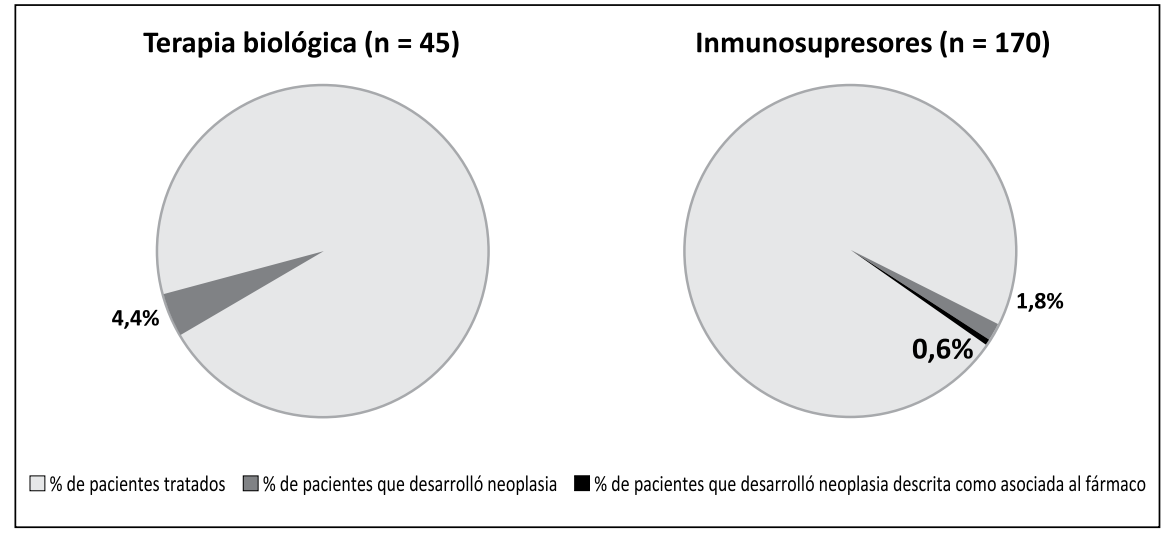

Figura 1. Desarrollo de neoplasia en pacientes con Ell tratados con inmunosupresores y terapia biológica. Destaca \% de neoplasias que se han descrito como relacionadas al uso de estos fármacos. con inmunosupresores (AZT), uno con terapia biológica (infliximab) (Tabla 2).

En relación al tratamiento para la EII, solamente en dos pacientes se modificó la terapia post-neoplasia. En uno de ellos de adalimumab a mesalazina y en el otro de AZT a mesalazina.

\section{Fármacos y neoplasia}

De los pacientes tratados con terapia biológica, 4,4\% había desarrollado algún tipo de neoplasia, ninguna de ellas descrita como asociada a estos fármacos. Un paciente con EC en monoterapia con infliximab por 3 años desarrolló un hipernefroma y el segundo paciente con CU desarrolló una LMC tras 5 años de monoterapia con adalimumab (previamente 7 años en tratamiento con AZT y metotrexato, los cuales habían sido suspendidos por alopecia e intolerancia gástrica, respectivamente). En el grupo de pacientes tratados con inmunosupresores, $1,8 \%$ de ellos había desarrollado una neoplasia, donde sólo una de ellas $(0,6 \%)$ se ha descrito como asociada al uso de estos fármacos (CPNM) (Figura 1).

\section{Discusión}

El desarrollo de neoplasia en pacientes con EII puede ser secundario a las alteraciones inmunológicas y al estado inflamatorio crónico presente en este grupo de pacientes, a los eventos adversos de las estrategias terapéuticas utilizadas (inmunosupresores y terapia biológica) o al riesgo de neoplasia descrito en la población general ${ }^{1,3,16,17}$.

En nuestro conocimiento, este es el primer estudio nacional que evalúa la presencia de neoplasia en una cohorte de paciente chilenos con EII, donde sólo un número pequeño de pacientes (10/437), equivalente a $2,3 \%$, han desarrollado algún tipo de neoplasia. Recientemente, un estudio prospectivo observacional con un seguimiento de 7 años señaló que 18 de los 590 pacientes (3\%) con EII desarrolló una neoplasia 6 .

Múltiples factores se han asociado al desarrollo de neoplasias en pacientes con EII, como edad al diagnóstico de la EII, años de duración de la enfermedad, manifestaciones extraintestinales asociadas, tabaco y exposición solar, este último factor en directa relación con el cáncer de piel ${ }^{1,6,18}$. Diversos estudios muestran que las neoplasias se desarrollan con mayor frecuencia en pacientes con diagnóstico de EII antes de los 40 años, lo cual fue observado en 9 de 10 pacientes de nuestro estudio ${ }^{6,18,19}$.

Estudios han mostrado que pacientes con EII tienen mayor riesgo de desarrollar CCR, con una prevalencia de $3,7 \%$ para $\mathrm{CU}$ y un riesgo relativo global de 2,5 para $\mathrm{EC}^{7,20}$. Sin embargo, estudios recientes señalan una frecuencia de CCR menor a la descrita previamente ${ }^{21}$. La historia familiar de CCR, menor edad al diagnóstico de EII, extensión y duración de la enfermedad, severidad de la inflamación y la presencia de CEP han sido señalados como factores de riesgo de $\mathrm{CCR}^{2}$. En nuestro estudio sólo dos pacientes con EII presentaron CCR, uno de ellos con una CU extensa de 30 años de evolución, con mala adherencia al tratamiento y a los controles endoscópicos. El segundo paciente tenía una EC de colon diagnosticada concomitantemente con el CCR a los 75 años. Distintos fármacos han sido asociados 
con la disminución en el riesgo de $\mathrm{CCR}^{2,22}$, dos meta-análisis recientes concluyeron que tanto la mesalazina como los tiopurínicos no tendría un rol en la prevención de $\mathrm{CCR}^{23,24}$.

El colangiocarcinoma se ha asociado a múltiples factores de riesgo, destacando la CEP y la EII ${ }^{4,25}$. Un estudio sueco que incluyó 604 pacientes, $79 \%$ con EII, demostró una frecuencia de colangiocarcinoma de $13 \%$ tras una mediana de seguimiento de 5,7 años $^{26}$. En nuestro estudio, una paciente con CU desarrolló este tipo de neoplasia presentando como factor de riesgo la presencia de CEP.

En pacientes con CU, la LMC se puede desarrollar con una frecuencia mayor a la población general, con un riesgo relativo de 1,5 a $2,9^{16}$. Recientemente, un estudio prospectivo que incluyó 19.486 pacientes con EII, mostró el desarrollo de enfermedades mieloides en 5 pacientes (dos leucemia aguda y tres un síndrome mielodisplásico). Dado que cuatro habían estado expuestos a tiopurínicos, los autores sugieren que este evento adverso debe ser considerado al momento de iniciar esta terapia ${ }^{27}$. En nuestro estudio, una paciente con CU desarrolló una LMC, estando en tratamiento con anti-TNF $\alpha$ por 5 años, y previo a ello 7 años con AZT y metotrexato los cuales habían sido suspendidos por eventos adversos.

El riesgo de neoplasia cervicouterina en pacientes con EII es aún controversial. Mientras algunos estudios han sugerido un aumento en el riesgo de neoplasia de cérvix ${ }^{28}$, otros han señalado que este no es diferente al compararlo con el grupo $\sin \mathrm{EII}^{29}$. Recientemente, un estudio que incluyó 27.408 mujeres con EII señaló que el riesgo de lesión intraepitelial de bajo y alto grado era mayor en pacientes con EII y el de neoplasia de cérvix estaba aumentado específicamente en pacientes con $\mathrm{EC}^{19}$. Además, se ha descrito un riesgo mayor asociado al uso de inmunosupresores Kane et al. demostraron que aquellas mujeres con EII en tratamiento inmunosupresor por tiempo mayor a 6 meses tenían mayor riesgo de presentar un frotis cervical alterado, con un odds ratio de $1,5^{28}$. En nuestro estudio, la paciente que desarrolló una neoplasia cervicouterina, sin embargo, se encontraba en tratamiento con mesalazina. Es posible que alteraciones inmunológicas presentes en la EII o el tratamiento con inmunosupresores puedan llevar a un mayor riesgo de neoplasia cervical dado el deterioro en la capacidad de eliminar el virus papiloma $^{30}$. Esto sugiere que pacientes con EII deben mantener un control de pesquisa de neoplasia cervical periódico y que el equipo médico debiera sugerir la vacuna para virus papiloma en mujeres entre los 9 y 26 años previo al inicio de la actividad sexual ${ }^{17}$.

El objetivo del tratamiento farmacológico en la EII es lograr la remisión de la enfermedad, junto con la curación de la mucosa intestinal. En segundo lugar, que esto se logre con la mayor seguridad y mínima toxicidad posible. Es un desafío evaluar el riesgo basal de desarrollo de neoplasia en pacientes con EII, debido a que la mayoría de los estudios han incluido pacientes que poseen una historia de exposición al menos a una clase de inmunosupresores, haciendo difícil separar el riesgo potencial por la enfermedad del riesgo por fármacos. En nuestro estudio, 4,4\% de los pacientes con terapia biológica y $1,7 \%$ de los usuarios de AZT desarrollaron alguna neoplasia (LMC e hipernefroma y CPNM, cáncer papilar de tiroides y seminoma, respectivamente). Sin embargo, sólo en un paciente el desarrollo de neoplasia estuvo relacionado con el tratamiento utilizado (AZT y CPNM). Existe evidencia que sugiere que el riesgo de cierto tipo de linfomas y cáncer de piel puede estar elevado en pacientes que reciben tiopurinas y terapia anti-TNF $\alpha$, particularmente cuando son utilizados en combinación ${ }^{16,18,27,31}$. Por otra parte, un estudio retrospectivo, que incluyó 185 pacientes con EII e historia de neoplasia, señaló que la exposición a terapia biológica y tiopurínicos posterior al diagnóstico de cáncer no estaba asociada a un aumento del riesgo de desarrollar una nueva neoplasia, al compararlos con aquellos pacientes con antecedentes de cáncer que no habían sido expuestos a estos fármacos ${ }^{32}$.

Evaluar la relación entre neoplasia y terapia anti-TNF $\alpha$ por sí misma es difícil, porque muy pocos pacientes han sido tratados con anti-TNF $\alpha$ sin actual o previa exposición a tiopurínicos. El uso de anti-TNF $\alpha$ ha sido asociado con melanoma, mientras no se ha encontrado una relación con el uso de tiopurínicos o mesalazina ${ }^{18}$. Recientemente, un estudio que incluyó 56.146 pacientes con EII y un metanálisis de 22 estudios controlados y distribuidos en forma aleatoria, concluyeron que la exposición a anti-TNF $\alpha$ en pacientes con EII no estaba asociada con un aumento en el riesgo de desarrollar neoplasias ${ }^{33,34}$. Sin embargo, no se puede excluir un aumento del riesgo asociado a mayor dosis acumulada y al período de seguimiento. 
A pesar de los avances en el tratamiento de la EII, las tiopurinas siguen siendo una estrategia útil en el manejo de estos pacientes, ya sea como monoterapia o asociados a terapia biológica ${ }^{8,9}$. Estos fármacos poseen un potencial mutagénico y carcinogénico que es relacionado con la dosis total y la duración del tratamiento, específicamente con el riesgo de enfermedades linfoproliferativas y de $\mathrm{CPNM}^{35-37}$. En nuestro registro, la paciente que desarrolló un CPNM estuvo con AZT en dosis adecuadas por 12 años debido a una CU de diagnóstico temprano (16 años), el cual fue suspendido y reemplazado por mesalazina al momento del diagnóstico de la neoplasia. Dada la clara asociación entre exposición a tiopurínicos y aumento en el riesgo de desarrollar CPNM es fundamental que estos pacientes tengan una disminución a la exposición al sol, uso de protector solar diario y control en dermatología ${ }^{17}$.

Dentro de las limitaciones de nuestro estudio consideramos que la frecuencia de neoplasias fue baja, por lo que no es posible estratificar según edad, tipo de neoplasia o terapia farmacológica. Además, su naturaleza retrospectiva limita obtener relaciones de causalidad.

En conclusión, considerando que en nuestra muestra sólo un pequeño grupo de pacientes con EII desarrolló neoplasia, el tratamiento indicado en estos pacientes debe estar determinado por la severidad de la enfermedad y no limitar las opciones terapéuticas por el temor a que desarrollen algún tipo de neoplasia. Es fundamental que el equipo médico y los pacientes sigan las recomendaciones con el fin de disminuir la posibilidad de desarrollar una neoplasia ya sea por la enfermedad en sí o como un evento adverso a la terapia.

\section{Referencias}

1. Kappelman MD, Farkas DK, Long MD, Erichsen R, Sandler RS, Sørensen HT, et al. Risk of cancer in patients with inflammatory bowel diseases: a nationwide population-based cohort study with 30 years of follow-up evaluation. Clin Gastroenterol Hepatol 2014; 12: 265-73.

2. Sebastián S, Hernández V, Myrelid P, Kariv R, Tsianos E, Toruner M, et al. Colorectal cancer in inflammatory bowel disease: results of the $3^{\text {rd }}$ ECCO pathogenesis scientific workshop (I). J Crohn's Colitis 2014; 8: 5-18.

3. Beaugerie L, Itzkowitz $\mathrm{SH}$. Cancers complicating inflammatory bowel disease. N Engl J Med 2015; 372: 1441-52.

4. Chang JS, Tsai CR, Chen LT. Medical risk factors associated with cholangiocarcinoma in Taiwan: A population-based case-control study. PLoS One 2013; 8 (7).

5. Egan L, D'Inca R, Jess T, Pellino G, Carbonnel F, Bokemeyer B, et al. Non-colorectal intestinal tract carcinomas in inflammatory bowel disease: Results of the 3rd ECCO Pathogenesis Scientific Workshop (II). J Crohns Colitis 2014; 8: 19-30.

6. Algaba A, Guerra I, Castaño A, De la Poza G, Castellano VM, López M, et al. Risk of cancer, with special reference to extra-intestinal malignancies, in patients with inflammatory bowel disease. World J Gastroenterol 2013; 19: 9359-65.

7. Canavan C, Abrams KR, Mayberry J. Meta-analysis: colorectal and small bowel cancer risk in patients with Crohn's disease. Aliment Pharmacol Ther 2006; 23: 1097-104.

8. Dignass A, Lindsay JO, Sturm A, Windsor A, Colombel JF, Allez M, et al. Second European evidence-based consensus on the diagnosis and management of ulcerative colitis part 2: current management. J Crohns Colitis 2012; 6: 991-1030.

9. Dignass A, Van Assche G, Lindsay JO, Lémann M, Söderholm J, Colombel JF, et al. The second European evidence-based Consensus on the diagnosis and management of Crohn's disease: Current management. J Crohns Colitis 2010; 4: 28-62.

10. McKenna MR, Stobaugh DJ, Deepak P. Melanoma and non-melanoma skin cancer in inflammatory bowel disease patients following tumor necrosis factor- $\alpha$ inhibitor monotherapy and in combination with thiopurines: analysis of the Food and Drug Administration Adverse Event Reporting System. J Gastrointestin Liver Dis 2014; 23: 267-71.

11. Targownik LE, Bernstein CN. Infectious and malignant complications of TNF inhibitor therapy in IBD. Am J Gastroenterol 2013; 108: 1835-42.

12. López-Martín C, Chaparro M, Espinosa L, Bejerano A, Maté J, Gisbert JP. Adverse events of thiopurine immunomodulators in patients with inflammatory bowel disease. Gastroenterol Hepatol 2011; 34: 385-92.

13. Dignass A, Eliakim R, Magro F, Maaser C, Chowers Y, Geboes K, et al. Second European evidence-based Consensus on the diagnosis and management of ulcerative colitis Part 1: Definitions and diagnosis (Spanish version)]. Rev Gastroenterol Mex 2014; 79 (4): 263-89.

14. Van Assche G, Dignass A, Panes J, Beaugerie L, Karagiannis J, Allez M, et al. European Crohn's and Colitis Organisation (ECCO). The second European eviden- 
ce-based Consensus on the diagnosis and management of Crohn's disease: Definitions and diagnosis. J Crohns Colitis 2010; 4 (1): 7-27.

15. Satsangi J, Silverberg MS, Vermeire S, Colombel JF. The Montreal classification of inflammatory bowel disease: controversies, consensus, and implications. Gut 2006; 55: 749-53.

16. Askling J, Brandt L. Lapidus A, Karlen P, Björkolm M, Löfberg R, et al. Risk of haematopoietic cancer in patients with inflammatory bowel disease. Gut 2005; 54: 617-22.

17. Magro F, Peyrin-Biroulet L, Sokol H, Aldeger X, Costa A, Higgins PD, et al. Extra-intestinal malignancies in inflammatory bowel disease: Results of the $3^{\text {rd }}$ ECCO Pathogenesis Scientific Workshop (III). J Crohns Colitis 2014; 8: 31-44.

18. Long MD, Martin CF, Pipkin CA, Herfarth HH, Sandler RS, Kappelman MD. Risk of melanoma and nonmelanoma skin cancer among patients with inflammatory bowel disease. Gastroenterology 2012; 143: 390-99.e1.

19. Rungoe C, Simonsen J, Riis L, Frisch M, Langholz E, Jess T. Inflammatory bowel disease and cervical neoplasia: A population-base nationwide cohort study. Clin Gastroenterol Hepatol 2014 [Epub ahead of print].

20. Eaden JA, Abrahms KR, Mayberry JF. The risk of colorectal cancer in ulcerative colitis: a meta-analysis. Gut 2001; 48: 526-35.

21. Beaugerie L, Svrcek M, Seksik P, Bouvier AM, Simon $\mathrm{T}$, Allez M, et al. Risk of colorectal high-grade dysplasia and cancer in a prospective observational cohort of patients with inflammatory bowel disease. Gastroenterology 2013; 145: 166-75.

22. Farraye FA, Odze RD, Eaden J, Itzkowitz SH. AGA technical review on the diagnosis and management of colorectal neoplasia in inflammatory bowel disease. Gastroenterology 2010; 138: 746-74.

23. Jess T, López A, Andersson M, Beaugerie L, Peyrin-Biroulet $\mathrm{L}$. Thiopurines and Risk of Colorectal Neoplasia in Patients With Inflammatory Bowel Disease: A Meta-analysis. Clin Gastroenterol Hepatol 2014; 12: 1793-800.

24. Nguyen GC, Gulamhusein A, Bernstein CN. 5-aminosalicylic acid is not protective against colorectal cancer in inflammatory bowel disease: a meta-analysis of non-referral populations. Am J Gastroenterol 2012; 107 : 1298-304.

25. Tyson GL, El-Serag HB. Risk factors of cholangiocarcinoma. Hepatology 2011; 54: 173-84.

26. Bergquist A, Ekbom A, Olsson R, Kornfeldt D, Lööf L, Danielsson A, et al. Hepatic and extrahepatic malignancies in primary sclerosing cholangitis. J Hepatol 2002; 36: 321-7.
27. López A, Mounier M, Bouvier AM, Carrat F, Maynadié $\mathrm{M}$, Beaugerie $\mathrm{L}$, et al. Increased risk of acute myeloid leukemias and myelodysplastic syndromes in patients who received thiopurine treatment for Inflammatory Bowel Disease. Clin Gastroenterol Hepatol 2014; 12: 1324-9.

28. Kane S, Khatibi B, Reddy D. Higher incidence of abnormal Pap smears in women with inflammatory bowel disease. Am J Gastroenterol 2008; 103: 631-6.

29. Lees CW, Critchley J, Chee N, Beez T, Gailer RE, Williams AR, et al. Lack of association between cervical dysplasia and IBD: a large case-control study. Inflamm Bowel Dis 2009; 15: 1621-9.

30. Einstein $\mathrm{MH}$, Schiller JT, Viscidi RP, Strickler HD, Coursaget $\mathrm{P}$, Tan T, et al. Clinician's guide to human papillomavirus immunology: knows and unknows. Lancet Infect 2009; 9: 347-56.

31. Singh S, Nagpal SJ, Murad MH, Yadav S, Kane SV, Pardi DS, et al. Inflammatory bowel disease is associated with an increased risk of melanoma: a systematic review and meta-analysis. Clin Gastroenterol Hepatol 2014; 12: 210-8.

32. Axelrad J, Bernheim O, Colombel J, Ananthakrishnan A, Yakjnik V, Katz S, et al. The risk of incident cancer in patients with Inflammatory Bowel Disease and a history of cancer following immunosuppression exposure. Inflamm Bowel Dis 2014; Suppl 20: S4-5.

33. Williams CJ, Peyrin-Biroulet L, Ford AC. Systematic review with meta-analysis: malignancies with anti-tumour necrosis factor-alpha therapy in inflammatory bowel disease. Aliment Pharmacol Ther 2014; 39: 447-58.

34. Andersen NN, Pasternak B, Basit S, Andersson M, Svanström H, Caspersen S, et al. Association between tumor necrosis factor-a antagonists and risk of cancer in patients with inflammatory bowel disease. JAMA 2014; 311: 2406-13.

35. Nguyen T, Vacek PM, O’Neill P, Colletti RB, Finette BA. Mutagenicity and potential carcinogenicity of thiopurine treatment in patients with inflammatory bowel disease. Cancer Res 2009; 69: 7004-12.

36. Beaugerie L, Brousse N, Bouier AM, Colombel JF, Lémann M, Cosnes J, et al. Lymphoproliferative disorders in patients receiving thiopurines for inflammatory bowel disease: a prospective observational cohort study. Lancet 2009; 374: 1617-25.

37. Peyrin-Biroulet L, Khosrotehrani K, Carrat F, Bouvier AM, Chevaux JB, Simon T, et al. Increased risk for nonmelanoma skin cancers in patients who received thiopurines for inflammatory bowel disease. Gastroenterology 2011; 141: 1621-8. 